\title{
Integrando tecnologias digitais na Educação de Jovens e Adultos: análise de publicações no Brasil
}

\author{
Leandro Paz ${ }^{1}$, Guilherme Weihmann ${ }^{1}$, Jessica Balbinot Plá ${ }^{1}$, Clevi Rapkiewicz ${ }^{1}$ \\ ${ }^{1}$ Colégio de Aplicação - Universidade Federal do Rio Grande do Sul (UFRGS) \\ \{leopazpoa,grweihmann,jessicab.pla\}@gmail.com, clevi@ufrgs.br
}

\begin{abstract}
Resumo: A inserção de tecnologias de informação e comunicação (TIC) na Educação de Jovens e Adultos (EJA) na atual sociedade é uma necessidade, uma vez que o público alvo dessa modalidade costuma ser de imigrantes digitais. Mas em que medida essa inserção tem ocorrido no Brasil? Visando analisar como está a integração de TIC na EJA no Brasil em termos de pesquisa este artigo analisa publicações organizadas num corpus composto dos artigos presentes em três revistas e dois anais de eventos de informática na educação entre os anos de 2009 e 2013 no Brasil. Foram identificadas lacunas na pesquisa para esse segmento educacional importante para a inclusão social e digital dos envolvidos.
\end{abstract}

Palavras-Chave: TIC; EJA; Mineração de Texto.

\begin{abstract}
Including Information and Communication Technologies (ICT) in the education of youth and adults in current society is a need, since the target audience of this modality is often comprised of digital immigrants. But to which degree such inclusion has been occurring in Brazil? In order to analyze the status of the integration between ICT and youth and adult education in Brazil, this paper analyzes publications assembled in a corpus composed of papers present in three journals and two conference proceedings of information technology in education between the years of 2009 and 2013. Several gaps were identified in the research of this important educational segment for the social and digital inclusion of the persons involved.
\end{abstract}

Keywords: ICT; Education of Youth and Adults; Text Mining.

\section{Introdução}

A Educação de Jovens Adultos (EJA) é um caso muito particular de educação nos desafios nacionais e globais atuais. A população atingida é composta por aqueles "que não puderam efetuar os estudos na idade regular, oportunidades educacionais apropriadas, consideradas as características do alunado, seus interesses, condições de vida e de trabalho, mediante cursos e exames. " (Brasil 2005, Art. 37) e fazem parte de uma necessidade global para o nosso presente século como justificado em relatório da UNESCO de 1996:

O conceito de educação ao longo da vida é a chave que abre as portas do século XXI; ele elimina a distinção tradicional entre educação formal inicial e educação permanente.(...) Ela deve abrir as possibilidades da educação a todos, com vários objetivos: oferecer uma segunda ou terceira oportunidade; dar resposta à sede de conhecimento, de beleza ou de superação de si mesmo; 
CBIE-LACLO 2015

Anais do XXI Workshop de Informática na Escola (WIE 2015)

ou, ainda, aprimorar e ampliar as formações estritamente associadas às exigências da vida profissional, incluindo as formações práticas. Em suma, a educação ao longo da vida, deve tirar proveito de todas as oportunidades oferecidas pela sociedade. (UNESCO, 1996)

Em um mundo onde a tecnologia está imperando, onde em quase todos os ambientes tem-se a presença de um celular ou computador, os jovens e adultos que não se formaram na idade padrão, estão atrelados ao paradigma não só da exclusão social como da exclusão digital. São imigrantes digitais, para Prensky (2011):

Os imigrantes digitais são os que chegaram à tecnologia digital mais tarde na vida e, por isso, precisaram se adaptar.](...) Muitos têm dificuldade em deixar antigos métodos para trás. Exemplos disso são imprimir e-mails ou não usar a internet como primeira fonte de informação. A distinção é mais cultural e de atitude.

O Prof. Dr. Pedro Demo situa Prensky na dicotomia entre as gerações mais novas terem certa aptidão com a informática e as gerações mais antigas terem pouca ou nenhuma aptidão com qualquer TIC. Segundo Prensky (2001 e 2006 apud DEMO, 2010):

(...) a criança - que é "nativa", enquanto nós, adultos, somos "imigrantes", [a criança] ao deparar-se com o computador, lida com ele sem saber ler, não precisando, ademais, de curso específico; ao contrário, fica aborrecida quando os pais (adultos) persistem em lhes dar "instruções". (p. 54)

Parte da população que caracteriza a EJA vem sofrendo alterações com o passar do tempo. Esta comunidade está sofrendo constantes alterações, está havendo um equilíbrio entre "imigrantes" e "nativos", com a inserção de alunos cada vez mais jovens na EJA, num fenômeno de juvenilização da EJA, o que já era apontado por Brunel em 2004.

Com o acesso a informação crescendo no Brasil, principalmente pelo uso da internet, que, não necessariamente precisa ser acessada através de um computador, é possível verificar que o uso da rede também não vem sendo aproveitado pelas faixas etárias mais avançadas. $\mathrm{O}$ que demonstra que há algum tipo de exclusão em relação às TIC, sejam elas computadores, celulares ou qualquer outra com a possibilidade de acessar a internet (Tabela 1).

Tabela 1: Dados CETIC - TIC Domicílios- Já acessaram Internet

\begin{tabular}{|l|c|c|c|c|c|}
\hline \multicolumn{1}{|c|}{2009} & \multicolumn{5}{|c|}{$\mathbf{2 0 1 3}$} \\
\hline \multicolumn{1}{|c|}{$\%$} & Sim & Não & Sim & Não & $\begin{array}{c}\text { Não sabe/não } \\
\text { respondeu }\end{array}$ \\
\hline De 10 a 15 anos & 77 & 23 & 85 & 15 & 0 \\
\hline De 16 a 24 anos & 83 & 17 & 87 & 13 & 0 \\
\hline De 25 a 34 anos & 65 & 35 & 75 & 25 & 0 \\
\hline De 35 a 44 anos & 38 & 62 & 56 & 44 & 0 \\
\hline De 45 a 59 anos & 22 & 78 & 38 & 62 & 0 \\
\hline De 60 anos ou mais & 8 & 92 & 14 & 86 & 0 \\
\hline
\end{tabular}


Certamente há exceções. Por exemplo, uma pessoa considerada idosa pode muito bem ter a "mente aberta" para as tecnologias disponíveis e querer aprender a usá-las. Em casa seu filho(a) ou neto(a) são os que podem inserir esta idosa no mundo digital, mas por falta de paciência ou até mesmo de interesse, algumas estes indivíduos passam a desencorajá-la, dizer-lhe que não é capaz de mexer em um computador. Isto é um exemplo dado, mas que ocorre frequentemente com pessoas, principalmente em uma faixa etária mais avançada, que querem entrar no mundo digital, mas tem dificuldade. É papel da escola ofertar oportunidades para essas pessoas. Mas que tipo de tecnologia digital, que tipo de uso tem sido feito na EJA no Brasil ? Buscando analisar esse universo coletou-se artigos de dois anais de eventos brasileiros e três revistas de Informática na Educação, de onde foram coletados 9 artigos relacionados a EJA, fonte na qual foi feita a pesquisa desse artigo, o qual está organizado em 4 seções além da presente introdução. $\mathrm{Na}$ seção 2, é apresentada a metodologia utilizada com dois momentos distintos: a mineração de artigos com uma explanação dos três softwares utilizados e seus métodos utilizados na mineração. Na seção 3, é apresentado o resultado da mineração dos 9 artigos relacionando a EJA e as TIC. Na última seção, a seção 4, são apresentadas algumas considerações finais.

\section{Metodologia:}

A análise foi feita com três mineradores distintos, cada um obtendo uma relação de 50 palavras para o estudo. A pesquisa relatada neste artigo seguiu os seguintes passos:

1. Levantamento bibliográfico referente a artigos relacionando as TIC e a EJA no período de 2009 a 2013. Foram escolhidas cinco publicações, sendo três revistas e dois anais de eventos relativos à informática na educação como pode ser observado na tabela 2 .

Tabela 2 - Artigos sobre TIC e EJA durante 5 anos

\begin{tabular}{|c|c|c|c|c|c|c|c|c|c|c|}
\hline & \multicolumn{2}{|c|}{$\begin{array}{c}\text { INFORMÁTICA } \\
\text { : TEORIA E } \\
\text { PRÁTICA }^{\mathrm{i}}\end{array}$} & \multicolumn{2}{|c|}{ RENOTE $^{\mathrm{ii}}$} & \multicolumn{2}{|c|}{$\mathbf{R B I E}^{\mathrm{iii}}$} & \multicolumn{2}{|c|}{$\mathbf{W I E}^{\mathrm{iv}}$} & \multicolumn{2}{|c|}{$\mathbf{S B I E}^{\mathrm{v}}$} \\
\hline Ano & $\begin{array}{l}\text { Total } \\
\text { de } \\
\text { Artigos }\end{array}$ & $\begin{array}{l}\text { Quantida } \\
\text { de de } \\
\text { artigos } \\
\text { do tema }\end{array}$ & $\begin{array}{l}\text { Total } \\
\text { de } \\
\text { Artigo } \\
\text { s }\end{array}$ & $\begin{array}{l}\text { Quanti } \\
\text { dade } \\
\text { de } \\
\text { artigos } \\
\text { do } \\
\text { tema }\end{array}$ & $\begin{array}{l}\text { Total } \\
\text { de } \\
\text { Artigo } \\
\text { s }\end{array}$ & $\begin{array}{l}\text { Quanti } \\
\text { dade } \\
\text { de } \\
\text { artigos } \\
\text { do } \\
\text { tema }\end{array}$ & $\begin{array}{l}\text { Total } \\
\text { de } \\
\text { Artigo } \\
\quad \text { s }\end{array}$ & $\begin{array}{l}\text { Quanti } \\
\text { dade } \\
\text { de } \\
\text { artigos } \\
\text { do } \\
\text { tema }\end{array}$ & $\begin{array}{l}\text { Total } \\
\text { de } \\
\text { Artig } \\
\text { os }\end{array}$ & $\begin{array}{l}\text { Quant } \\
\text { idade } \\
\text { de } \\
\text { artigo } \\
\text { s do } \\
\text { tema }\end{array}$ \\
\hline 2009 & 31 & 0 & 117 & 1 & 16 & 0 & 44 & 0 & 83 & 0 \\
\hline 2010 & 23 & 1 & 107 & 2 & 18 & 0 & 60 & 1 & 132 & 0 \\
\hline 2011 & 22 & 0 & 120 & 0 & 19 & 1 & 71 & 0 & 138 & 1 \\
\hline 2012 & 27 & 0 & 114 & 0 & 27 & 0 & 47 & 0 & 130 & 0 \\
\hline 2013 & 25 & 0 & 139 & 1 & 30 & 1 & 49 & 0 & 101 & 0 \\
\hline Total & 128 & 1 & 597 & 4 & 110 & 2 & 271 & 1 & 584 & 1 \\
\hline Corpus & & & & & 9 & igos & & & & \\
\hline
\end{tabular}


2. Montagem de um corpus para análise contendo todos os artigos selecionados. O corpus é uma compilação de textos, ou seja, a junção de todos os textos coletados para o estudo. O corpus em questão é a junção de 9 artigos relativos a EJA e as TIC, apontados na Tabela 2.

3. Mineração desse corpus utilizando os softwares TextAnalyzer ${ }^{\mathrm{vi}}$, Sobek ${ }^{\mathrm{vii}} \mathrm{e}$ Wordle $^{\text {viii }}$.

Mineração de texto tem como uma de suas finalidades na educação ser um método utilizado para extrair informações de textos, capturando as suas tendências (AZEVEDO; BEHAR; REATEGUI, 2011). Nos processos de mineração utilizados no corpus podemse obter os resultados na forma de tabela, grafo e nuvem de palavras, conforme o minerador utilizado. Fornecidos pelos softwares TextAnalyzer, Sobek e Wordle respectivamente. Alguns mineradores tem a funcionalidade de excluir as stopwords do estudo. As stopwords normalmente são palavras de composição textual que não contribuem com o estudo (artigos, preposições, numerais).

O TextAnalyzer é um software online gratuito que permite encontrar desde as frases frequentes, frequências das palavras, até o número de palavras e caracteres utilizados no texto, fazendo o uso de dados estatísticos para o estudo em questão. A partir deste levantamento inicial, é possível verificar a quantidade de palavras presentes no texto entre outros tipos de levantamento. Também é possível trabalhar com textos em várias línguas diferentes. No processo de mineração pelo TextAnalyzer não há como escolher as "stopwords". Para suprir esta necessidade, foram selecionadas as 122 palavras mais frequentes, onde 72 palavras eram "stopwords" e as outras 50 foram utilizadas no estudo.

O Sobek é um software desenvolvido por uma equipe multidisciplinar da Universidade Federal do Rio Grande do Sul. Este software é gratuito, executável com diferentes sistemas operacionais tais como Mac, Linux e Windows, e o seu download pode ser feito no http://sobek.ufrgs.br. Seu objetivo é relacionar os termos mais utilizados em um texto, resultando em um grafo. $\mathrm{O}$ grafo terá as informações mais relevantes no texto minerado conectadas umas às outras. Selecionando um termo do grafo, o Sobek informa quantas vezes este termo aparece no texto e em que frases ele está, facilitando a análise.

$\mathrm{Na}$ formação de grafo pelo Sobek foram relacionadas 50 palavras, porém foram excluídas a sigla "http" e a palavra "Paulo" que não estão relacionadas ao estudo.

O Wordle é uma ferramenta online que permite obter uma nuvem das palavras mais utilizadas no corpus em análise, podendo-se escolher quantas palavras mostram-se adequadas para o estudo, definir o layout, a direção das palavras, enfim, editar a nuvem da maneira que seja útil para o estudo. $O$ tamanho da palavra na nuvem está diretamente relacionado com a sua frequência no texto. Com o Wordle, formou-se uma nuvem também com 50 palavras. Esta ferramenta tem o recurso das "stopwords", porém, só se podem excluir estas palavras em uma língua, ou seja, se excluirmos as "stopwords" que estão em português, as que estão no texto em outras línguas permanecerão na nuvem. E foi isto que ocorreu, das 50 palavras que compõem a nuvem, 4 são "stopwords" em inglês. São elas: of, the, and e in. Houve um caso de repetição de palavras (por uma estar com A maiúsculo e a outra não) e um nome pessoal, são as palavras "Alunos", "Educação" e "Paulo". Sendo assim, a nuvem foi editada a fim de excluir estas palavras e deixar as 42 restantes. 


\section{Resultados}

O primeiro resultado que apontamos é a indicação de quais foram os instrumentos de TIC mencionados nos artigos (Tabela 3 ).

Tabela 3 - Instrumentos de TIC mencionados

\begin{tabular}{|c|c|c|c|c|}
\hline Software & Freq. & Aplicação & Idioma & Online? \\
\hline Facebook & 2 & $\begin{array}{l}\text { Compartilhamento de produções; } \\
\text { integração com o Moodle }\end{array}$ & Português & Sim \\
\hline Moodle & 1 & Integração com Facebook e Twitter & Português & \\
\hline $\begin{array}{l}\text { Objetos de } \\
\text { Aprendizagem } \\
\text { (diversos) }\end{array}$ & 2 & $\begin{array}{l}\text { Matemática, informática; aplicação } \\
\text { avaliativa com educandos }\end{array}$ & Português & Não \\
\hline $\begin{array}{l}\text { Paint } \\
\text { (Windows) }\end{array}$ & 1 & $\begin{array}{l}\text { Estudo de formas básicas em } \\
\text { geografia plana }\end{array}$ & Português & Não \\
\hline Prezi & 1 & Criação de infográficos & Português & Não \\
\hline SIAI & 1 & $\begin{array}{l}\text { Sistema web de suporte a tutoriais; } \\
\text { sequenciador de }\end{array}$ & Português & Sim \\
\hline Twitter & 2 & $\begin{array}{l}\text { Compartilhamento de produções; } \\
\text { integração com o Moodle }\end{array}$ & Português & $\operatorname{Sim}$ \\
\hline XHOME3D & 1 & $\begin{array}{l}\text { Estudo de geometria plana criando } \\
\text { plantas baixas }\end{array}$ & Inglês & Não \\
\hline $\begin{array}{l}\text { Sem menção } \\
\text { direta }\end{array}$ & 3 & $\begin{array}{l}3 \text { estudos sem menção direta a } \\
\text { algum software }\end{array}$ & - & - \\
\hline
\end{tabular}

Há uma variedade de softwares mencionados nos artigos. Na maior parte dos estudos, não houve estudo de caso com estudantes, apenas projetos e sugestões metodológicas a respeito do uso dos softwares. Alguns se destacam, no entanto, com aplicação testada em sala de aula, como: alguns dos vários Objetos de Aprendizagem mencionados, com os quais se buscou aferir a eficácia de uso com turmas de EJA, tanto para ensino de conteúdo como para construir habilidades com as TIC. Destaque também para o editor de apresentações Prezi, em conjunto com Facebook e Twitter para compartilhar produções; e o uso do Paint e do XHome3D para estudar geometria plana. A partir desses dados, destacamos: a predominância em buscar abordar tópicos do ensino de matemática, a tendência de utilizar softwares traduzidos para o português, e a baixa quantidade de serviços online utilizados, indicando que a computação em nuvem ainda não parece ser uma realidade para aplicação na EJA..

Com o objetivo de refletir acerca da localização das pesquisas que envolvem TICs e EJA, realizamos um mapeamento - Figura 1 - contabilizando em quantos artigos cada instituição é mencionada, a partir da informação de localidade na afiliação dos autores de cada artigo. É possível perceber uma grande concentração da produção no Rio Grande do Sul, mais especificamente nas instituições: UFRGS, PUC-RS - 1, UNISINOS - 2, UFSM -1 . 
Figura 1: Mapeamento de localidade institucional de autores dos artigos do corpus

Na Tabela 4 apresentamos o resultado das análises dos artigos, utilizando o corpus com recursos de mineração online TextAnalyzer, Wordle (nuvem) e Sobek respectivamente:

Tabela 4 - Mineração da ferramenta online TextAnalyzer

\begin{tabular}{|c|c|c|c|c|c|c|c|c|}
\hline \multicolumn{2}{|c|}{ Termos } & $\begin{array}{l}\text { Qua } \\
\text { ntid } \\
\text { ade }\end{array}$ & \multicolumn{2}{|c|}{ Termos } & $\begin{array}{l}\text { Qua } \\
\text { ntid } \\
\text { ade }\end{array}$ & \multicolumn{2}{|c|}{ Termos } & $\begin{array}{c}\text { Qua } \\
\text { ntid } \\
\text { ade }\end{array}$ \\
\hline 1. & Educação & 268 & 18 & figura & 84 & 35 & Diferentes & 58 \\
\hline 2. & Alunos & 259 & 19 & computador & 82 & 36 & Pesquisa & 56 \\
\hline 3. & aprendizagem & 161 & 20 & integração & 74 & 37 & Conteúdo & 56 \\
\hline 4. & forma & 130 & 21 & processo & 74 & 38 & estudantes & 54 \\
\hline 5. & mouse & 129 & 22 & pessoas & 70 & 39 & Software & 53 \\
\hline 6. & uso & 128 & 23 & novas & 68 & 40 & Dados & 53 \\
\hline 7. & ensino & 109 & 24 & redes & 67 & 41 & utilização & 52 \\
\hline 8. & moodle & 108 & 25 & atividade & 67 & 42 & Leitura & 52 \\
\hline 9. & adultos & 102 & 26 & social & 65 & 43 & dificuldades & 52 \\
\hline 10. & conhecimento & 97 & 27 & informática & 65 & 44 & Idade & 51 \\
\hline 11. & trabalho & 90 & 28 & escola & 64 & 45 & Projeto & 51 \\
\hline 12. & jovens & 90 & 29 & aluno & 62 & 46 & Agente & 51 \\
\hline 13. & sociais & 88 & 30 & grupo & 62 & 47 & Curso & 50 \\
\hline 14. & atividades & 87 & 31 & sites & 61 & 48 & professores & 49 \\
\hline 15. & eja & 86 & 32 & digital & 59 & 49 & Texto & 46 \\
\hline 16. & tecnologias & 85 & 33 & acesso & 59 & 50 & facebook & 46 \\
\hline 17. & objetos & 84 & 34 & aplicação & 58 & & & \\
\hline
\end{tabular}

Através do TextAnalyzer, a relação das TIC com a EJA fora destacada em três linhas (palavras grifadas). Palavras grifadas em amarelo tem o significado do saber, de como decorre a atividade, no caso, ocorre interação dos alunos com seus colegas e professor, e com as TIC. Os alunos realizam leituras online, e fazem atividades aplicadas a eles em diferentes plataformas. Estas plataformas fazem parte do grupo de palavras grifadas em verde, que são palavras relacionadas às TIC. 
É apontado que os alunos da EJA estão realizando atividades com foco na utilização do mouse, atividades através da plataforma Moodle e do Facebook, seja através de textos ou de atividades para entregar. A dificuldade com o mouse, inclusive, é destacada em Hollewerger, Almeida e Doll (2010), destacando vários problemas como uso excessivo de força e tensão ou segurar o mouse com a ponta dos dedos. Notar que essas dificuldades estão associadas a idade, palavra grifada em vermelho.

A massa de dados textual pode ser melhor visualizada na nuvem gerada através do Wordle, apresentada na Figura 2.

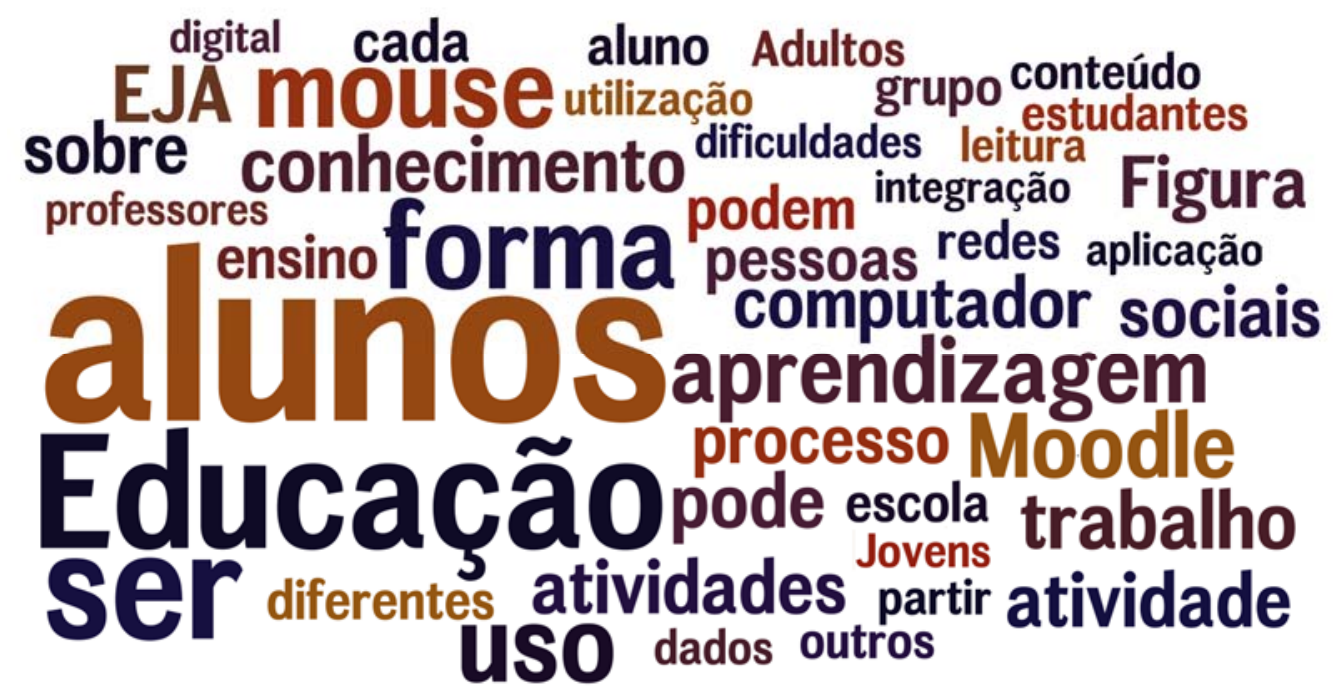

Figura 2: Nuvem gerada pela ferramenta online Wordle

A EJA está sendo inserida nas Tecnologias de Informação e Comunicação através de plataformas como, por exemplo, o Moodle e redes sociais, plataformas que ajudam na integração dos alunos. Também realizam leituras utilizando o computador e, como ferramentas de aprendizagem e mediadas pelos seus professores.

Apesar da leitura imagética propiciada pela nuvem, não é possível observar a relação entre as palavras. Assim, o recurso da visualização através de um grafo, propiciado pelo Sobek, torna-se pertinente. Conforme já mencionado na metodologia, das 50 palavras estabelecidas para análise duas foram retiradas, restando 48 apresentadas nas Figuras 3 e 4 . Na Figura 4 podem ser observadas as relações entre as palavras e na Figura 3 aquelas que em termos de frequência apareceram na mineração, mas sem relação direta com o grafo principal (Figura 4).

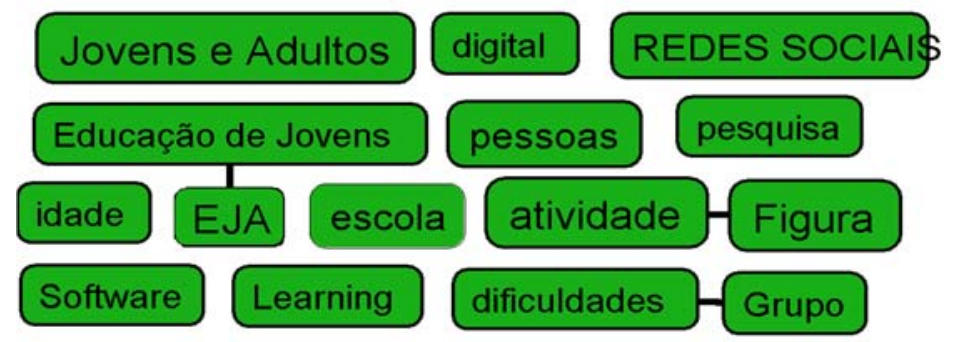

Figura 3: Palavras que ficaram sem ligação com grafo principal do Sobek, porém, muitas relacionadas com a temática em análise 


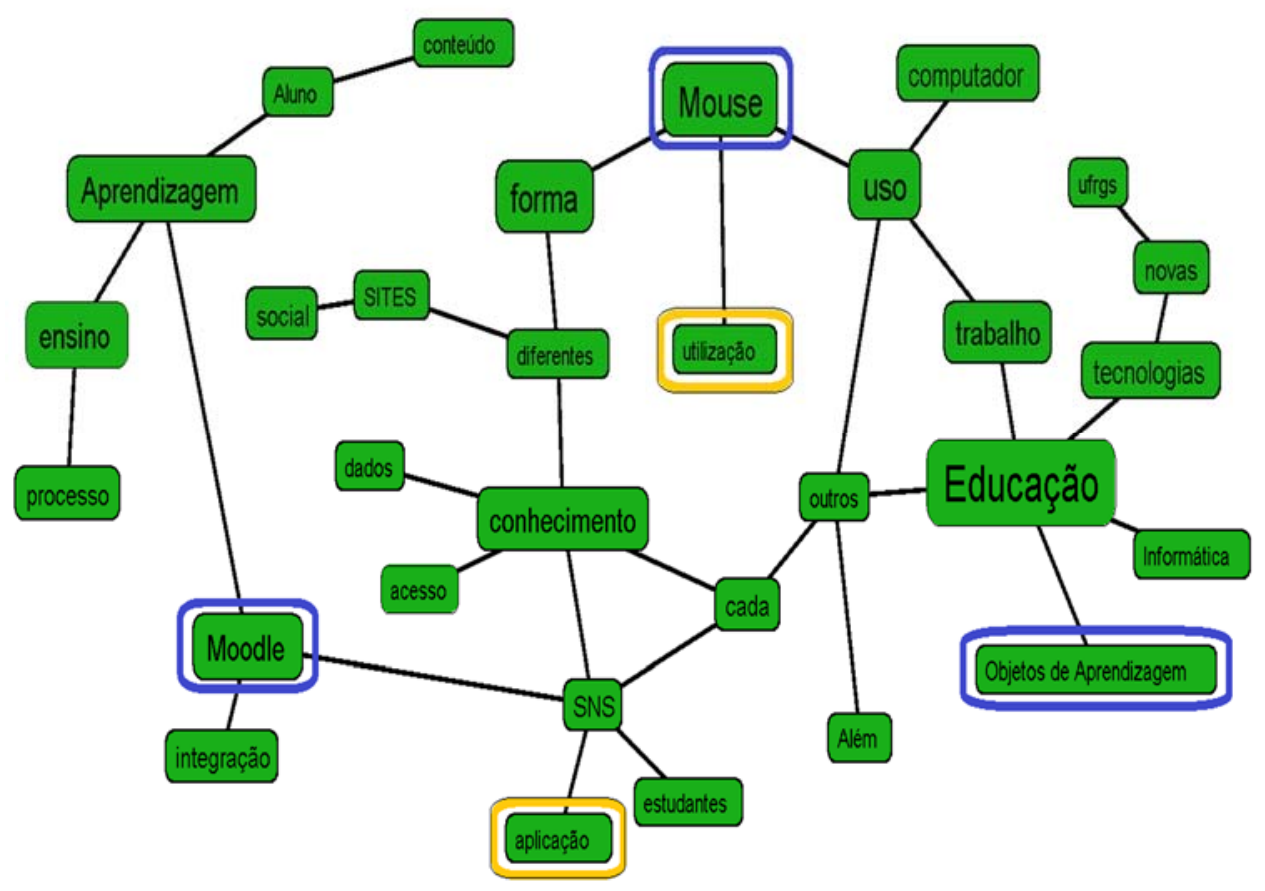

Figura 4: Grafo gerado pela ferramenta Sobek;

A EJA tem como atividades com as TIC, o suporte do mouse, atividades no Moodle estimulando o ensino com atividades nesta plataforma e interagindo com seus colegas através de fóruns e bate-papos. Conforme apontado anteriormente na mineração com o TextAnalyzer, palavras grifadas em amarelo estão relacionadas com o saber, modo de aplicação da atividade. As palavras grifadas em azul estão relacionadas às TIC.

Através de comentários minerados com o Sobek, o Moodle, além de estar sendo utilizado sozinho, há interação com, sites de redes sociais como Facebook e Twitter, conforme apontado por exemplo em Braz (2012). No que se refere a utilização de objetos de aprendizagem são apontados alguns trabalhos visaram a análise de aceitação destes recursos pelos alunos da EJA.

Um dos maiores desafios dos imigrantes digitais é o uso do mouse, mexer neste hardware acaba sendo um desafio, pois os alunos da EJA chegam a ter medo de utilizálo, mexem nele com receio de que algo ruim pode acontecer. Porém, seu uso é muito mais profundo do que apenas ir para a pasta que se deseja, ajuda a entender o funcionamento do computador e também o pensamento cognitivo. Isto se torna claro neste trecho extraído de um dos componentes do corpus:

(...) deve-se reservar tempo suficiente com exercícios específicos para a utilização do mouse, pois a capacidade de utilização do periférico, influencia diretamente o avanço da aquisição de conhecimentos em relação ao computador. Para se obter um avanço na utilização do mouse podem ser utilizados programas de desenho como o Paint (Windows), Tux Paint (Linux), ou também programas específicos para o treinamento do mouse, que permitem aos alunos a exploração do movimento e a precisão no 
CBIE-LACLO 2015

Anais do XXI Workshop de Informática na Escola (WIE 2015)

manejo do mouse. (HOLLERWEGER, L., ALMEIDA, S. T., DOLL, J., 2010, p.176)

Com estas extrações é possível confrontar os resultados da análise de corpus com as observações finais feitas por Buratto ao questionar-se sobre o foco do professor na aulas de informática para EJA. Segundo BURATTO (2010): “A preocupação principal [dos professores] é incluí-los digitalmente como forma de construir autonomia, primando pela constante motivação, afim de mantê-los nos cursos. ". E isto é provado também através de um esclarecimento de PRENSKY (2011):

(...) se os professores quiserem ajudar os alunos do século 21 a aprender. Alguns acham que a pedagogia vai mudar automaticamente, assim que os "nativos digitais" se tornarem professores. Eu discordo. Há pressões forçando os professores novos a adotar métodos antigos. Nós precisamos fazer um grande esforço de mudança. Primeiro, mudar a forma como nós ensinamos --nossas pedagogias. Depois, mudar a tecnologia que nos dá suporte. Finalmente, mudar o que nós ensinamos --nosso currículo-- para estarmos em acordo com o contexto e as necessidades do século 21.

\section{Considerações finais}

A análise resultante das minerações obtidas através destes três softwares, nos permite extrair algumas conclusões e também deixa espaço para algumas dúvidas quanto as TIC na EJA. É notável a utilização de plataformas interativas como o Moodle ou redes sociais como Facebook para a realização de atividades como, por exemplo, uma leitura de um texto qualquer, onde após a leitura, o usuário expressa a sua opinião e confronta ou corrobora com a opinião de seus colegas e professor. Estas plataformas unidas a uma atividade onde é necessária a expressão de opinião, ou uma resposta, são agentes facilitadores para a inclusão digital e social dos indivíduos que as utilizam, pois acabam interagindo digitalmente e socialmente.

Outra atividade que vem sendo executada com frequência é a competência no uso do mouse, visando desmistificar este hardware perante aos alunos. Alunos de idade mais avançada chegam a ter medo do seu uso. Por isso a alta demanda que esta atividade tem na EJA. Como visto nas tabelas 1 e 2, quanto maior a faixa etária dos indivíduos, menor é o uso de uma TIC ou de um recurso de TIC pelo indivíduo. Isto corrobora com a mineração realizada através do TextAnalyzer, onde temos como uma das possíveis dificuldades de implantação de TIC na EJA, a idade.

Sugerimos a continuidade desse estudo através da busca de artigos presentes em outras revistas e eventos com ênfase na EJA, não especificada de informática na educação, de forma a ampliar os estudos iniciados.

\section{Referências Bibliográficas:}

AZEVEDO, B.F.T; BEHAR, P.A; REATEGUI, E. B. Aplicação da Mineração de Textos na Área de Educação. Educação e Tecnologia - Um percurso interinstitucional. Editora Essentia. 2011. 
BRAZ, L. M. Uma Arquitetura Flexível para Promover Integração entre o Moodle e Sites de Redes Sociais. in TCC - Orientador Prof. Dr. Sérgio Crespo C. S. Pinto. 2012. UNISINOS.

BRUNEL, C. Jovens cada vez mais jovens na educação de jovens e adultos. Porto Alegre: Mediação, 2004.

BURATTO, D. B. Para o que (e porque) atentar em aulas de Informática para adultos da EJA. in TCC - Orientador Johannes Doll. 2011. UFRGS

CETIC. TIC Domicílios e Usuários, 2009. Disponível em $<$ http://www.cetic.br/tics/usuarios/2009/total-brasil/>. Acesso em 20 de abril de 2015.

CETIC. TIC Domicílios e Usuários, 2013. Disponível em $<$ http://www.cetic.br/tics/usuarios/2013/total-brasil/>. Acesso em 20 de abril de 2015.

DEMO, P. Aprendizagens e Novas Tecnologias. Revista Brasileira de Docência, Ensino e Pesquisa em Educação Física - Vol. 1, n. 1, p.53-75, Agosto/2009.

GOMES, P. Leia entrevista do autor da expressão 'imigrantes digitais' - Veja os principais trechos da entrevista de Mark Prensky, autor que cunhou as expressões "nativos" e "imigrantes digitais". Folha.com. 2011. Acesso em 12 de maio de 2015 em:

http://www.marcprensky.com/international/Leia $\% 20$ entrevista $\% 20 \mathrm{do} \% 20$ autor $\% 20 \mathrm{da} \%$ 20expressao\%20imigrantes $\% 20$ digitais.pdf

HOLLERWEGER, L., ALMEIDA, S. T., DOLL, J. Adultos maduros e informática: o mouse no caminho. Informática na Educação: Teoria \& Prática, v.13, n.1, 2010, p 167 179.

PRENSKY, M. Digital natives, digital immigrants. On the Horizon MCB University Press, v.9, n. 5, 2001.

UNESCO. Learning: the treasure within; report to UNESCO of the International Commission on Education for the Twenty-first Century (highlights). Paris: UNESCO, 1996. Publicado pelo Setor de Educação da Representação da UNESCO no Brasil em 2010.

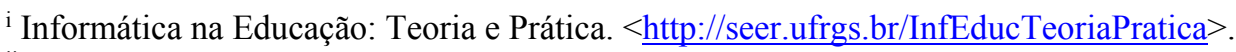

${ }^{i i}$ Renote - Revista Novas Tecnologias na Educação. <http://seer.ufrgs.br/renote>.

iii RBIE - Revista Brasileira de Informática na Educação. <http://www.br-ie.org/index.php/rbie>.

${ }^{\text {iv }}$ WIE - Workshop de Informática na Educação. < $<$ http://www.br-ie.org/index.php/anaiswie $>$.

${ }^{v}$ SBIE - Simpósio Brasileiro de Informática na Educação. < http://www.br-ie.org/index.php/anaissbie $>$.

viTextAnalyzer disponível em: $<$ http://www.online-utility.org/text/analyzer.jsp $>$. Acesso: Outubro 2014.

vii Sobek disponível em: $<$ http://sobek.ufrgs.br/>. Acesso em: Outubro 2014.

viii Wordle disponível em: $<$ http://www.wordle.net/>. Acesso em: Outubro 2014.

*WIE e SBIE - artigos coletados de 2009 a 2013.
} 